\title{
Children's Interactions in an Asynchronous Video Mediated Communication Environment
}

\author{
Michail N. Giannakos ${ }^{1}$, Konstantinos Chorianopoulos ${ }^{1}$, Paul Johns ${ }^{2}$, \\ Kori Inkpen ${ }^{2}$, and Honglu $\mathrm{Du}^{3}$ \\ ${ }^{1}$ Ionian University, Corfu, Greece \\ \{mgiannak, choko\} aionio.gr \\ ${ }^{2}$ Microsoft Research, Redmond, WA, USA \\ \{paul.johns, kori\} amicrosoft.com \\ ${ }^{3}$ Penn State University, PA, USA \\ hzd106@ist.psu. edu
}

\begin{abstract}
Video-mediated communication (VMC) has become a feasible way to connect people in remote places for work and play. Nevertheless, little research has been done with regard to children and VMC. In this paper, we explore the behavior of a group of children, who exchanged video messages in an informal context. In particular, we have analyzed 386 videos over a period of 11 weeks, which were exchanged by 30 students of $4^{\text {th }}$ and $5^{\text {th }}$ grade from USA and Greece. We found that the number of views and the duration of a video message significantly depend on the gender of the viewer and creator. Most notably, girls created more messages, but boys viewed their own messages more. Finally, there are video messages with numerous views, which indicates that some videos have content qualities beyond the communication message itself. Overall, the practical implications of these findings indicate that the developers of asynchronous VMC should consider functionalities for preserving some of the video messages.
\end{abstract}

Keywords: Asynchronous, Video-Mediated Communication, Children, Thread, Gender.

\section{Video Mediated Communication}

Computer-mediated communication (CMC) includes a variety of electronic messages and audio-video systems. There is also increasing evidence that CMC mediums is replacing traditional forms of media and becoming a primary mode of communication in the workplace [1]. In this research, we are exploring the potential of CMC in the classroom, with a special focus on intercultural communication between distant places. Several researchers $[2,3]$ have claimed text-based asynchronous CMC to be incoherent for reasons such as the lack of simultaneous feedback, and the disrupted turn adjacency. However, video mediated communication (VMC) provides social context cues such as non-verbal signals (facial expressions, gestures); paraverbal cues (voice volume); and interpersonal cues (gender, physical appearance). 
Various studies have shown that children are usually ineffective communicators because they have not mastered the necessary linguistic or cognitive competencies [4]. Bruner asserted that a language-based medium like email would be more complex for children than a medium that leveraged actions, bodily movement, or imagery. VMC is considered the most desirable to support nonverbal communication among children [5].

Few studies have reported gender differences in CMC as a main interest (independent variable). Hiltz and Johnson [6] found that females viewed CMC more favorably than males. Another notable research with an intra-organizational mail system, females believed e-mail to be easier to use, more effective and efficient than males [7]. Furthermore, Adrianson [8] mentions that females tended to produce more messages than males in the face-to-face communication, but in the CMC there was no significant difference. Another useful variable which characterizes discussion or/and conversation is the depth and the breadth. In our study we used the depth and the breadth of a thread (conversation) to explore the size of each conversation/discussion.

The current study focuses on using asynchronous video messages for children's communication. We evaluated a video-based asynchronous tool called Video Pal [9]. We were particularly interested in asynchronous video because it is an ideal tool to support communication between people from different parts of the world spanning many time-zones.

\section{Methodology}

The sample of participants in this study was comprised of 30 students. From the total of the participants, 25 pupils ( 12 boys and 13 girls) were from USA and 5 pupils ( 3 boys and 2 girls) were from Greece, as such 15 were boys (50\%) and 15 (50\%) girls.

One tradition in the USA partner school is that every year, each grade selects a country to study, and learns about that country's culture and lifestyles. In the final week of the fall semester, the children give presentations about what they have learned about that country to all the teachers, students and their parents. From the perspective of Greek pupils their key motivations was that it was regarded as a good opportunity for them to practice their English and it also enabled them to learn more about computers.

They employed the VideoPal user interface (figure 1), which has a main window and a message window. The main window allows users to quickly see which conversation threads are available, the properties of each thread (e.g., number of messages, number of unread messages in the thread), a visual presentation of one conversation, the new messages which are shown at bottom of the visualization panel, and the current users' profile photo. From this main window, users can create a new video or play an existing video message. The message window serves two purposes - to record a new message and play a received message. Users can play a received message, record a video message, and play a video preview, which is listed in the up right corner of this window. 

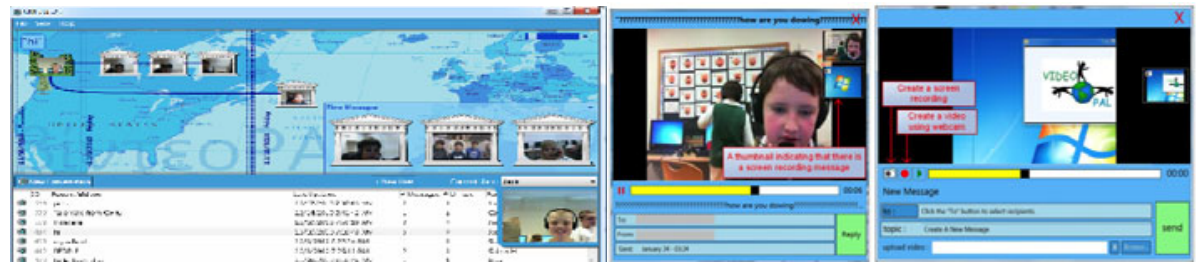

Fig. 1. Video Pal main window (left), message view/creation windows (right)

The duration of the study was approximately eleven weeks. The study began on the $12^{\text {th }}$ of November (2010) and finished on the $28^{\text {th }}$ of February (2011) with breaks during Christmas (17 Dec- 11Jan) and 04-15 Feb. The following graph (figure 2) indicates the number of videos created each week and their length during the study. During the first week each country-group only sent videos internally, in order to get familiar with the user interface.

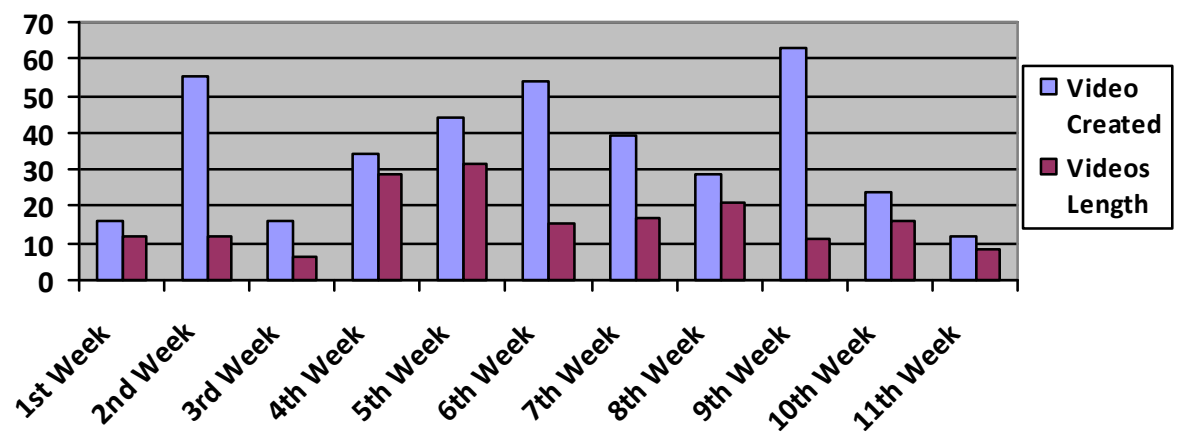

Fig. 2. The number of videos created each week and the average length of the videos each week

This paper presents an analysis of the computer log data. Descriptive data on all the video messages transmitted during the study were obtained through computer log files. In particular, we examined the following interactions: 1) number of author viewings, 2) number of recipient viewings, 3) video duration, 4) number of recipients and 5) distance of a video from the start of the thread (Video Distance). Authors' viewings refer to the number of times the author of video replayed the video after sending it. Recipient viewings is the number of times a recipient viewed the video. Video duration is the length of each video in seconds. Number of recipients is the number of recipients per video message. The following table (table 1) outlines the descriptive statistics of the videos.

In general, key dimensions of communication are depth and breadth of the discussion-conversation $[10,11,12]$. As such, we used the video thread depth and the breadth in order to analyze the video discussions. Using VideoPal, the children could either reply to an existing video, or start a new thread/conversation. There were 171 threads created during this study. The mean number of videos in a thread was 2.26 with S.D.=1.11. 
Table 1. Descriptive statistics of the videos $(\mathrm{N}=386)$

\begin{tabular}{|l|l|c|c|l|c|c|}
\hline & Country & No & $\%$ & Gender & No & $\%$ \\
\hline Author & GR & 84 & 21.8 & Male & 156 & 40.4 \\
& USA & 302 & 78.2 & Female & 230 & 59.6 \\
\hline Recipient(s) & GR & 127 & 32.9 & Male & 110 & 28.5 \\
& USA & 139 & 36.0 & Female & 154 & 39.9 \\
& Both & 120 & 31.1 & Both & 122 & 31.6 \\
\hline & \multicolumn{7}{|c|}{ Mean (S.D.) } \\
\hline Number of recipients & \multicolumn{7}{|c|}{$17.98(3.26)$} \\
\hline Video Viewings & Author & \multicolumn{7}{|c|}{$1.93(1.13(1.19)$} \\
\hline Video Duration (sec) & \multicolumn{7}{|c|}{ Recipient(s) } & $6.26(9.77)$ \\
\hline Video Distance & \multicolumn{7}{|c|}{ (1.23) } \\
\hline
\end{tabular}

In our context, we defined the depth of a thread as the length of the longest chain in the thread. The breadth of the thread is the maximum number of replies to any video in the thread (figure 3).

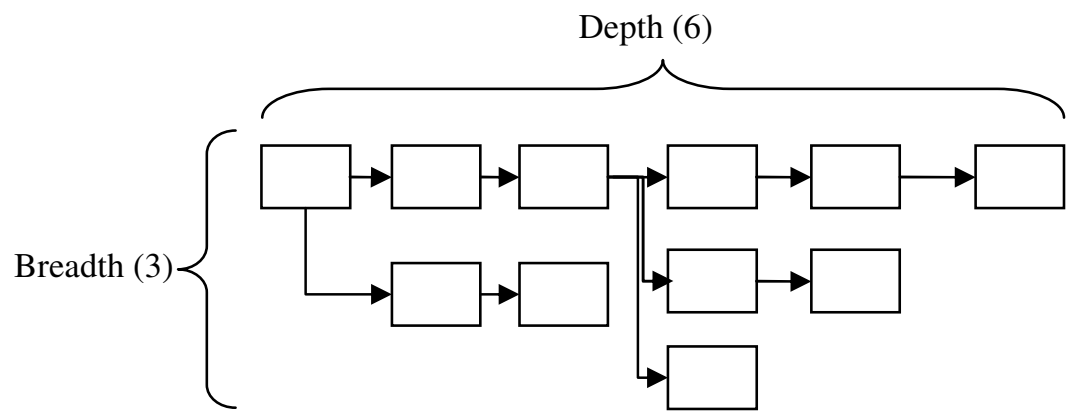

Fig. 3. An example of a thread depth and breadth

\section{Research Findings}

T-tests were conducted in order to investigate possible differences. T-test method was chosen, because it applies to the problem of estimating means [13]. Firstly we explored the impact of the gender of the pupil-author on number of viewings and the length of the video.

Using a t-test the following scores were deduced (table 2). The results showed that $\mathrm{t}(212)=3.51, \mathrm{p}<0.01$ and $\mathrm{t}(293)=4.04, \mathrm{p}<0.001$. This indicated that the number of viewings and the duration of the videos were significantly different between male and female authors. This leads us to the result that male authors view their videos more times and produce longer videos than female authors. Although females created shorter video messages, they also tended to create more video messages $(70 \%$ more, as shown in table 1 , although this difference was not statistically significant based on t-test result). 
Table 2. Testing the impact of gender in authors viewings and video duration

\begin{tabular}{|l|c|c|c|c|c|c|c|c|}
\hline & \multicolumn{2}{|c|}{ Male } & \multicolumn{2}{c|}{ Female } & \multicolumn{3}{c|}{ t-test } & Results \\
\cline { 2 - 9 } & Mean & S.D & Mean & S.D & $\mathrm{t}$ & $\mathrm{df}$ & Sig. & \\
\hline $\begin{array}{l}\text { Author No of } \\
\text { viewings }\end{array}$ & 1.11 & 1.82 & 0.55 & 0.95 & 3.51 & 212 & $.001 * *$ & $\begin{array}{l}\text { Significant } \\
\text { Difference }\end{array}$ \\
\hline Video duration & 20.97 & 16.39 & 14.53 & 13.75 & 4.04 & 293 & $.000 * * *$ & $\begin{array}{l}\text { Significant } \\
\text { Difference }\end{array}$ \\
\hline $\begin{array}{l}\text { Recipients no } \\
\text { of viewings }\end{array}$ & 3.72 & 4.18 & 3.23 & 4.34 & .91 & 240 & .361 & $\begin{array}{c}\text { No } \\
\text { Significant } \\
\text { Difference }\end{array}$ \\
\hline$* * * \mathrm{p}<0.001$ & $* * \mathrm{p}<0.01$ & \multicolumn{7}{|c|}{$* \mathrm{p}<0.05$} \\
\hline
\end{tabular}

We also explored the impact of recipients' gender on the number of viewings. Using a t-test of two groups the following scores were deduced (table 2). The results showed that $\mathrm{t}(240)=0.91, \mathrm{p}>0.05$. This indicated that the number of times a recipient viewed a video was not statistically different for males and females.

As we noticed, the gender of the participants often had a significant effect on their interactions with the videos. The following figure (figure 4) shows the influence of gender and on pupils' viewings.
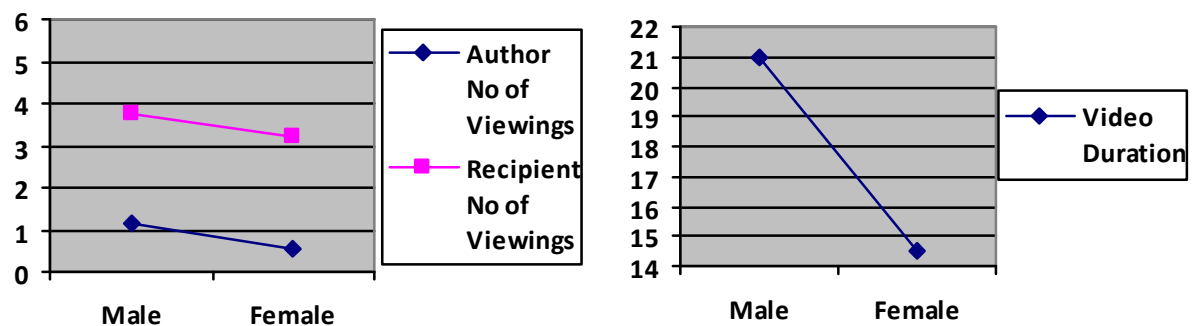

Fig. 4. The Influence of gender on user viewings and video duration (seconds)

We used Pearson's correlation coefficient between the features of the VMC, to quantify the strength of the relationship between the features. This analysis suggests that some of the features are related. For example, the number of recipients' viewings is strongly correlated with the number of recipients' and the distance of the video from the start of the thread (negatively). The duration of the video has strong negative correlation with the distance of the video from the start of the thread. Moreover, authors' number of viewings was significantly correlated with the number of recipients' viewings. All the correlations between the features are indicated in table 3 .

Since the breadth and depth of the thread are two key dimensions of the conversation we chose to examine their behavior. However, an analysis of breadth and depth in small threads is not trustworthy, therefore, we made a depth-breadth analysis in threads, which were longer than 4 in depth (in total 8 threads). Figure 5 shows that as the breadth of the conversation grew, so did the depth. 
Table 3. Pearson's Correlation Coefficient between VMC features

\begin{tabular}{|c|c|c|c|c|c|}
\hline $\begin{array}{c}\text { VMC } \\
\text { Features }\end{array}$ & $\begin{array}{c}\text { Authors No of } \\
\text { viewings }\end{array}$ & $\begin{array}{c}\text { Recipients No } \\
\text { of viewings }\end{array}$ & $\begin{array}{c}\text { Video } \\
\text { duration }\end{array}$ & $\begin{array}{l}\text { Recipients } \\
\text { Number }\end{array}$ & Dvsth \\
\hline $\begin{array}{l}\text { Authors No of } \\
\text { viewings }\end{array}$ & $\begin{array}{l}1.000 \\
(386)\end{array}$ & & & & \\
\hline $\begin{array}{l}\text { Recipients No } \\
\text { of viewings }\end{array}$ & $\begin{array}{l}.125^{*} \\
(386)\end{array}$ & $\begin{array}{l}1.000 \\
(386)\end{array}$ & & & \\
\hline $\begin{array}{l}\text { Video } \\
\text { duration }\end{array}$ & $\begin{array}{l}.021 \\
(386)\end{array}$ & $\begin{array}{l}.063 \\
(386)\end{array}$ & $\begin{array}{l}1.000 \\
(386)\end{array}$ & & \\
\hline $\begin{array}{l}\text { Recipients } \\
\text { Number }\end{array}$ & $\begin{array}{c}.019 \\
(319) \\
\end{array}$ & $\begin{array}{l}.561 * * \\
(319)\end{array}$ & $\begin{array}{l}-.017 \\
(319)\end{array}$ & $\begin{array}{l}1.000 \\
(319)\end{array}$ & \\
\hline Dvsth & $\begin{array}{l}-.086 \\
(386)\end{array}$ & $\begin{array}{l}-.072 * \\
(386) \\
\end{array}$ & $\begin{array}{c}-.244 * * \\
(386)\end{array}$ & $\begin{array}{l}-.026 \\
(319)\end{array}$ & $\begin{array}{l}1.000 \\
(386) \\
\end{array}$ \\
\hline
\end{tabular}

* Correlation is significant at the 0.05 level. ** Correlation is significant at the 0.01 level.

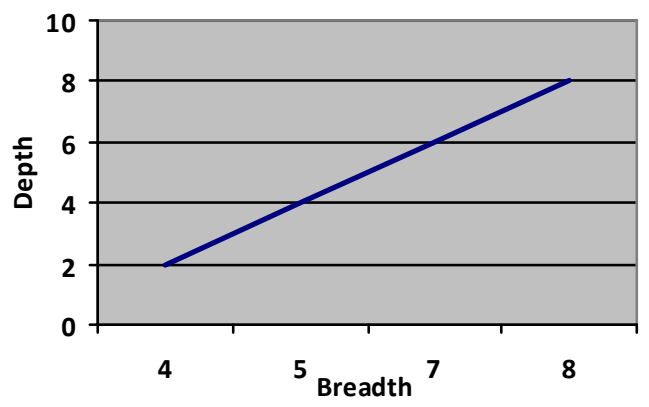

Fig. 5. Representation of breadth and depth behavior

\section{Discussion and Ongoing Work}

The above findings provide early evidence on VMC among children. In particular, the findings indicate that female video authors produce approximately $70 \%$ more videos than male authors. This finding is consistent with Hiltz and Johnson [6] results which found that females believed $\mathrm{CMC}$ to be easier to use, more effective and efficient than males. Moreover, Allen [7] states that females viewed CMC more favorably than males. However, Adrianson [8] found that females tended to produce more messages than males in face to face communication, but in CMC there was no significant difference. Therefore, this finding is not consistent with Tarasuik et al., [14] which states that for very young children video communication can have many of the same effects as a physical communication.

On the other hand, our findings indicate that males are viewing more of their produced videos than females; however, we found that females produce more videos. This may suggest that females prefer more active roles (produce) than males (view) in 
VMC $[15,16]$. In addition, females prefer to be more expressive at sending and decoding nonverbal messages and participate in more non-verbal communication behavior [17]. Interestingly, the findings indicate that males produce significant lengthier videos than females. This is consistent with Sussman and Tyson [18] which found that males produce longer postings in cyber-conversation.

The Pearson's correlation coefficients demonstrate that video duration has a strong negative correlation with the distance of the video from the start of the thread. This is means that long duration videos are more likely at the beginning of a thread and short duration videos are most likely at the end of a thread. However this result may arise, at least in part, because video near the end of a thread indicate a communication flow, this communication flow led the participants in specific subjects and as a result in shorter videos. This is enforced by the fact that video based communication increased the feeling of "connectedness" between the participants [19].

Another interesting finding was that the number of authors' viewings was significantly related to the number of recipients' viewings and the number of recipients' viewings was significantly related to video depth. This result may arise from the nature of some videos; we can assume that the relation comes from the interest in some videos. However, we watched the most famous (high visibility of both authors and recipients site) videos and we realized that all these videos included useful personal information and the majority of them belong to a long depth videos. Interestingly, we observed these videos and we conclude that they were either introductory or descriptive for a participant. For instance, all these videos provided information such as, favorite foods, music, singer and hobby of the author; on the other hand videos with low visibility contain just questions. In brief, video messages with questions do not have repeat views, but video messages with statements can get a lot of attention, both by the creator and the recipients.

In an online context Parks and Floyd [11] and Parks and Roberts [12] found CMC users exhibit high levels of breadth and depth and form close relationships with their online partners. In our study we observed that the two key dimensions of each communication environment (depth and breadth) are following an absolute analogy behavior. This indicates that a video conversation keeps the analogies in their dimensions and they are not growing lop-sided.

As with any study, there are some limitations. For example, this pilot study was performed over a short time and the number of participants was quite small. Moreover, there was an asymmetry in the number of children between Greece and USA. We would also like to validate and extend the current findings with qualitative studies, such as longitudinal observations, or diary studies and investigate other challenges in cross-cultural VMC between children. Moreover, the follow up study would draw from a wider and more symmetric sample of children between the countries. In the light of an internal and external analysis of the groups we will identify which effects are caused from the culture of each group and which not. Finally, a deeper analysis of the video-user interactions using information such as timestamp of each view and video content analysis will provide a step ahead toward the understanding of children video mediated communication. 


\section{References}

1. Tassabehji, R., Vakola, M.: Business email: The killer impact. Communications of the ACM 48(11), 64-70 (2005)

2. Herring, S.C.: Interactional coherence in CMC. Journal of Computer-Mediated Communication 4(4) (1999), http: / / jcmc.indiana. edu/vol4/issue4/herring.html

3. Marcoccia, M.: On-line polylogues: conversation structure and participation framework in Internet newsgroups. Journal of Pragmatics 36(1), 115-145 (2004)

4. Piaget, J.: The language and thought of the child. Brace \& Company, Inc., NY (1926)

5. Ballagas, R., Kaye, J., Ames, M., Go, J., Raffle, H.: Family communication: phone conversations with children. In: Interaction Design for Children 2009, pp. 321-324 (2009)

6. Hiltz, R.S., Johnson, K.: User satisfaction with computer-mediated communication systems. Management Science 36, 739-751 (1990)

7. Allen, B.J.: Gender and computer-mediated communication. Sex-Roles 32(7/8), 557-563 (1995)

8. Adrianson, L.: Gender and computer-mediated communication: Group processes in problem solving. Computers in Human Behavior 17(1), 71-94 (2001)

9. Du, H., Inkpen, K.M., Tang, J., Roseway, A., Hoff, A., Johns, P., Czerwinski, M., Meyers, B., Chorianopoulos, K., Gross, T., Lungstrang, P.: Video Pal: An Asynchronous VideoBased Communication System to Connect Children from US and Greece. In: Proc. of CSCW. ACM Press, New York (2011)

10. Gay, G., Lentini, M.: Use of communication resources in a networked collaborative design environment. Journal of Computer-Mediated Communication 1(1) (1995)

11. Parks, M.R., Floyd, K.: Making friends in cyperspace. Journal of Computer-Mediated Communication 46, 80-97 (1996)

12. Parks, M.R., Roberts, L.D.: 'Making MOOsic': The development of personal relationships on-line and a comparison to their off-line counterparts. Journal of Social and Personal Relationships 15, 517-537 (1998)

13. Pallant, J.: T-tests. In: Pallant, J. (ed.) SPSS. Survival manual, 2nd edn., pp. 140-159. Open University Press, Berkshire (2005)

14. Tarasuik, J.C., Galligan, R., Kaufman, J.: Almost Being There: Video Communication with Young Children. PLoS ONE 6(2), e17129 (2011), doi:10.1371/journal.pone.0017129

15. Herring, S.: Gender differences in computer mediated communication: Bringing familiar baggage to the new frontier. Paper presented at the American Library Association Annual Convention, Miami, FL (June 1994)

16. Spender, D.: Nattering on the net: Women, power and cyberspace. Spinifex Press Ltd., North Melbourne (1995)

17. Briton, N.J., Hall, J.: Beliefs about female and male nonverbal communication. Sex Roles 23, 79-90 (1995)

18. Sussman, N., Tyson, D.: Sex and power: gender differences in computer mediated interactions. Computers in Human Behavior 16(4), 381-394 (2000)

19. Zuckerman, O., Maes, P.: Awareness system for children in distributed families. In: Ext. Abst. of IDC. ACM Press, New York (2004) 